\title{
GRAM MATRIX ASSOCIATED TO CONTROLLED FRAMES
}

\author{
E. OSGOOEI AND A. RAHIMI
}

\begin{abstract}
Controlled frames have been recently introduced in Hilbert spaces to improve the numerical efficiency of interactive algorithms for inverting the frame operator. In this paper, unlike the cross-Gram matrix of two different sequences which is not always a diagnostic tool, we define the controlled-Gram matrix of a sequence as a practical implement to diagnose that a given sequence is a controlled Bessel, frame or Riesz basis. Also, we discuss the cases that the operator associated to controlled Gram matrix will be bounded, invertible, Hilbert-Schmidt or a trace-class operator. Similar to standard frames, we present an explicit structure for controlled Riesz bases and show that every $(U, C)$-controlled Riesz basis $\left\{f_{k}\right\}_{k=1}^{\infty}$ is in the form $\left\{U^{-1} C M e_{k}\right\}_{k=1}^{\infty}$, where $M$ is a bijective operator on $H$. Furthermore, we propose an equivalent accessible condition to the sequence $\left\{f_{k}\right\}_{k=1}^{\infty}$ being a $(U, C)$-controlled Riesz basis.
\end{abstract}

\section{INTRODUCTION}

Frames in Hilbert spaces were first introduced by Duffin and Schaeffer to deal with nonharmonic Fourier series in 1952 [9] and widely studied from 1986 since the great work by Daubechies, Grossmann and Meyer constructed [8]. Nowadays frames play an important role in pure and applied mathematics, also have many applications in signal processing 10, coding and communications 20, filter bank theory [5]. We refer to [6, 7, for an introduction to frame theory and its applications.

Controlled frames as a generalization of frames, have been introduced for getting an improved solution of a linear system of equation $A x=B$, which this system can be solved by equation $P A x=P B$, where $P$ is a suitable matrix to get a better duplicate algorithm [4. Controlled frames used earlier just as a tool for spherical wavelets and the relation between controlled frames and standard frames were developed in [3]. The main advantage of these frames lies in the fact that they retain all the advantages of standard frames but additionally they give a generalized way to check the frame condition while offering a numerical advantage in the sense of preconditioning. Recent developments in this direction can be found in [11, 12, 14, 17, 18, 19] and the references therein.

A sequence $\left\{f_{k}\right\}_{k \in I} \subseteq H$ is a frame for $H$ if there exist $0<A \leq B<\infty$ such that

$$
A\|f\|^{2} \leq \sum_{k \in I}\left|\left\langle f, f_{k}\right\rangle\right|^{2} \leq B\|f\|^{2}, \quad f \in H .
$$

The constants $A$ and $B$ are called lower and upper frame bounds, respectively. The sequence $\left\{f_{k}\right\}_{k \in I} \subseteq H$ is a Bessel sequence for $H$, if the right hand inequality in

2010 Mathematics Subject Classification. 42C15; 42C40; 94A12; 68M10.

Key words and phrases. controlled frames; Gram matrix; Gram operators; controlled Riesz bases. 
(1.1), holds for all $f \in H$.

Let $\left\{f_{k}\right\}_{k \in I}$ be a Bessel sequence for $H$. Then the operator

$$
T: \ell^{2}(\mathbb{N}) \rightarrow H, \quad T\left\{c_{k}\right\}_{k \in I}=\sum_{k \in I} c_{k} f_{k},
$$

is called the synthesis operator and its adjoint

$$
T^{*}: H \rightarrow \ell^{2}(\mathbb{N}), \quad T^{*} f=\left\{\left\langle f, f_{k}\right\rangle\right\}_{k \in I},
$$

is called the analysis operator of $\left\{f_{k}\right\}_{k \in I}$. By composing the operators $T$ and $T^{*}$, we get the frame operator $S=T T^{*}$, which is a bounded, positive, invertible operator and $A I \leq S \leq B I$.

A Riesz basis for $H$ is a family of the form $\left\{U e_{k}\right\}_{k=1}^{\infty}$, where $\left\{e_{k}\right\}_{k=1}^{\infty}$ is an orthonormal basis for $H$ and $U$ is a bounded bijective operator on $H$.

Checking equation (1.1) is not always an easy task in practice. So the conditions for a sequences $\left\{f_{k}\right\}_{k \in I}$ being a Bessel sequence, frame, or Riesz basis can be expressed in terms of the so-called Gram matrix.

1.1. Gram matrix of discrete frames. If $\left\{f_{k}\right\}_{k=1}^{\infty}$ is a Bessel sequence, we can compose the synthesis operator $T$ and its adjoint $T^{*}$ to obtain the bounded operator

$$
T^{*} T: \ell^{2}(\mathbb{N}) \rightarrow \ell^{2}(\mathbb{N}) ; \quad T^{*} T\left\{c_{k}\right\}_{k=1}^{\infty}=\left\{\left\langle\sum_{\ell=1}^{\infty} c_{\ell} f_{\ell}, f_{k}\right\rangle\right\}_{k=1}^{\infty} .
$$

This operator is called the Gram operator on $\ell^{2}(\mathbb{N})$ associated to $\left\{f_{k}\right\}_{k=1}^{\infty}$ and corresponds to a matrix given by

$$
T^{*} T=\left\{\left\langle f_{k}, f_{j}\right\rangle\right\}_{j, k=1}^{\infty} .
$$

The matrix $\left\{\left\langle f_{k}, f_{j}\right\rangle\right\}_{j, k=1}^{\infty}$ is called the matrix associated with $\left\{f_{k}\right\}_{k=1}^{\infty}$ or Gram matrix.

The ability of combining the synthesis and analysis operators of a Bessel sequence to make a sensitive operator is essential in frame theory and its applications. For example in [15, for given two different Bessel sequences $\left\{f_{k}\right\}_{k \in I}$ and $\left\{g_{k}\right\}_{k \in I}$ the synthesis operator of $\left\{f_{k}\right\}_{k \in I}$ with the analysis operator of $\left\{g_{k}\right\}_{k \in I}$ is composed and a fundamental operator is generated. This operator is called the cross-Gram operator associated with the sequence $\left\{\left\langle f_{k}, g_{j}\right\rangle\right\}_{j, k=1}^{\infty}$ [2, 16, and the conditions that this operator be well-defined bounded or invertible is studied.

In this paper, we introduce the Gram operator of controlled frames as a practical tool and discuss the cases in which this operator can be well-defined, bounded, Hilbert-Schmidt, trace class, compact and invertible.

The content of this paper is as follows: In Section 2, the Gram operator and Gram matrix of $(U, C)$-controlled frames introduced and a practical method to diagnose Bessel sequence is given by the concept of controlled Gram matrix. Also a bounded operator from $\ell^{1}(\mathbb{N})$ to $\ell^{\infty}(\mathbb{N})$ is achieved with the assumption that the controlled Gram operator is well-defined and bounded on $\ell^{2}(\mathbb{N})$. In Section 3 , the general construction of $(U, C)$-controlled Riesz bases proposed and an equivalent feasible method for $\left\{f_{k}\right\}_{k=1}^{\infty}$ to being a $(U, C)$-controlled Riesz basis, given. Throughout this paper, $H$ is a separable Hilbert space and $G L(H)$ is the space of all bounded and invertible operators on $H$ and $G L^{+}(H)$ is the space of all bounded, invertible and positive operators on $H$. Also $U, C \in G L(H)$. 


\section{Gram matrix of CONTROLled FRAMES}

Controlled frames with one and two controller operators were first introduced in [3] and [14, respectively. They are equivalent to standard frames and so this concept gives a generalization way to check the frame conditions.

Definition 2.1. Let $\left\{f_{k}\right\}_{k \in I}$ be a sequence of vectors in a Hilbert space $\mathcal{H}$ and $U, C \in G L(\mathcal{H})$. Then $\left\{f_{k}\right\}_{k \in I}$ is called a frame controlled by $U$ and $C$ or $(U, C)$ controlled frame if there exist two constants $0<A \leq B<\infty$, such that

$$
A\|f\|^{2} \leq \sum_{k \in I}\left\langle f, U f_{k}\right\rangle\left\langle C f_{k}, f\right\rangle \leq B\|f\|^{2}, \quad f \in \mathcal{H} .
$$

If only the right inequality holds, then we call $\left\{f_{k}\right\}_{k \in I}$ a $(U, C)$-controlled Bessel sequence. If $A=B$ then $\left\{f_{k}\right\}_{k \in I}$ is called a $(U, C)$-controlled tight frame.

Let $F=\left\{f_{k}\right\}_{k \in I}$ be a Bessel sequence of elements in $H$. We define the synthesis operator $T_{U F}$,

$$
T_{U F}: \ell^{2}(I) \rightarrow H, \quad T_{U F}\left(\left\{a_{k}\right\}_{k \in I}\right)=\sum_{k \in I} a_{k} U f_{k}, \quad\left\{a_{k}\right\}_{k \in I} \in \ell^{2}(I),
$$

and the adjoint operator $T_{U F}^{*}$ which is called the analysis operator is as follows:

$$
T_{U F}^{*}: H \rightarrow \ell^{2}(I), \quad T_{U F}^{*}=\left\{\left\langle f, U f_{k}\right\rangle\right\}_{k \in I}
$$

Now we define the controlled frame operator $S_{U C}$ on $H$

$$
S_{U C} f=T_{C F} T_{U F}^{*} f=\sum_{k \in I}\left\langle f, U f_{k}\right\rangle C f_{k}, \quad f \in H .
$$

It is easy to see that if $F=\left\{f_{k}\right\}_{k \in I}$ is a $(U, C)$-controlled frame with bounds $A_{U C}$ and $B_{U C}$, then $S_{U C}$ is well-defined and

$$
A_{U C} I d_{H} \leq S_{U C} \leq B_{U C} I d_{H} .
$$

Hence $S_{U C}$ is a bounded, invertible, self-adjoint and positive linear operator. Therefore, we have $S_{U C}=S_{U C}^{*}=S_{C U}[3$, 14].

Proposition 2.2. 14 Let $U, C \in G L(H)$ and $F$ be a family of vectors in a Hilbert space $H$. Then the following statements hold:

(1) If $F$ is a $(U, C)$-controlled frame for $H$. Then $F$ is a frame for $H$.

(2) If $F$ is a frame for $H$ and $C S_{F} U^{*}$ is a positive operator, then $F$ is a $(U, C)$ controlled frame for $H$.

By the above proposition for a frame $F$ which is also a $(U, C)$-controlled frame for $H$, we have

$$
C S_{F} U^{*}=S_{U C}=S_{U C}^{*}=U S_{F} C^{*}=S_{C U}
$$

Also we have new reconstruction formula as follows:

$$
f=\sum_{i \in I}\left\langle f, U f_{i}\right\rangle S_{U C}^{-1} C f_{i}=\sum_{i \in I}\left\langle f, S_{U C}^{-1} U f_{i}\right\rangle C f_{i}, \quad f \in H .
$$

Proposition 2.3. 6] Let $T: \mathcal{H} \rightarrow \mathcal{H}$ be a linear operator. Then the following conditions are equivalent:

(1) There exist $A>0$ and $B<\infty$, such that $A I \leq T \leq B I$;

(2) $T$ is positive and there exist $A>0$ and $B<\infty$, such that $A\|f\|^{2} \leq$ $\left\|T^{\frac{1}{2}} f\right\|^{2} \leq B\|f\|^{2}$ 
(3) $T$ is positive and $T^{\frac{1}{2}} \in G L(\mathcal{H})$.

(4) There exists a self-adjoint operator $S \in G L(\mathcal{H})$, such that $S^{2}=T$;

(5) $T \in G L^{(+)}(\mathcal{H})$;

(6) There exist constants $A>0$ and $B<\infty$ and an operator $C \in G L^{(+)}(\mathcal{H})$ such that $A C \leq T \leq B C$;

(7) For every $C \in G L^{(+)}(\mathcal{H})$, there exist constants $A>0$ and $B<\infty$ such that $A C \leq T \leq B C$.

Since controlled frames and standard frames are equivalent in some cases, we define the Gram matrix of controlled frames as an effective tool to diagnose the controlled Bessel, frame or Riesz bases. But as we see the results are not always the same as cross-Gram matrix or Gram matrix of standard frames.

If $\left\{f_{k}\right\}_{k=1}^{\infty}$ is a $(U, C)$-controlled Bessel sequence, we can compose the synthesis operator $T_{C F}$ and $T_{U F}^{*}$, so we obtain the bounded operator

$$
T_{U F}^{*} T_{C F}: \ell^{2}(\mathbb{N}) \rightarrow \ell^{2}(\mathbb{N}), \quad T_{U F}^{*} T_{C F}\left\{a_{k}\right\}_{k=1}^{\infty}=\left\{\left\langle\sum_{j=1}^{\infty} a_{j} C f_{j}, U f_{k}\right\rangle\right\}_{k=1}^{\infty} .
$$

We call this operator the $(U, C)$-controlled Gram operator.

Suppose that $\left\{e_{k}\right\}_{k=1}^{\infty}$ is the canonical orthonormal basis for $\ell^{2}(\mathbb{N})$, the $j k$-th entry in the matrix representation of $T_{U F}^{*} T_{C F}$ is

$$
T_{U F}^{*} T_{C F}=\left\{\left\langle C f_{j}, U f_{k}\right\rangle\right\}_{k, j=1}^{\infty} .
$$

The matrix $\left\{\left\langle C f_{j}, U f_{k}\right\rangle\right\}_{k, j=1}^{\infty}$ is called the Gram matrix associated to $(U, C)$ controlled Bessel sequence $\left\{f_{k}\right\}_{k=1}^{\infty}$ or $(U, C)$-controlled Gram matrix associated to $\left\{f_{k}\right\}_{k=1}^{\infty}$.

Remark 2.4. The above argument shows that if $\left\{f_{k}\right\}_{k=1}^{\infty}$ is a $(U, C)$-controlled Bessel sequence, the $(U, C)$-controlled Gram matrix associated to $\left\{f_{k}\right\}_{k=1}^{\infty}$ is well-defined and bounded.

Example 2.5. Let $\left\{e_{k}\right\}_{k=1}^{\infty}$ be the canonical orthonormal basis for $\ell^{2}(\mathbb{N})$. Consider the sequence $f_{2 k+1}=e_{2 k+1}-e_{2 k+2}, k=0,1,2, \ldots$ and $f_{2 k}=e_{2 k-1}+e_{2 k}, k=$ $1,2,3, \ldots$. If we define the operators

$$
C: \ell^{2}(\mathbb{N}) \rightarrow \ell^{2}(\mathbb{N}), \quad C\left(x_{1}, x_{2}, x_{3}, x_{4}, \ldots\right)=\left(-x_{1}, x_{2},-x_{3}, x_{4}, \ldots\right)
$$

and

$$
U: \ell^{2}(\mathbb{N}) \rightarrow \ell^{2}(\mathbb{N}), \quad U\left(x_{1}, x_{2}, x_{3}, x_{4}, \ldots\right)=\left(x_{1},-x_{2}, x_{3},-x_{4}, \ldots\right) .
$$

Then a straight calculation shows that $\left\{f_{k}\right\}_{k=1}^{\infty}$ is a $(U, C)$-controlled tight frame for $\ell^{2}(\mathbb{N})$ with bound 2 and the $(U, C)$-controlled Gram matrix associated to $\left\{\left\langle C f_{k}, U f_{j}\right\rangle\right\}_{j, k=1}^{\infty}$ is well-defined and bounded on $\ell^{2}(\mathbb{N})$ with bound 2 .

In Example 2.1 of [15, we saw that although the cross-Gram matrix associated to $\left\{\left\langle f_{k}, g_{j}\right\rangle\right\}_{j, k=1}^{\infty}$ is well-defined and bounded, $\left\{f_{k}\right\}_{k=1}^{\infty}$ is not a Bessel sequence. Now a logical question is that: can we say the sequence $\left\{f_{k}\right\}_{k=1}^{\infty}$ is Bessel if the $(U, C)$-controlled Gram matrix associated to $\left\{f_{k}\right\}_{k=1}^{\infty}$ is well-defined and bounded? The following lemma shows that the answer is positive.

Lemma 2.6. Suppose that $U, C \in G L(H)$ and the $(U, C)$-controlled Gram matrix associated to $\left\{f_{k}\right\}_{k=1}^{\infty}$ is well-defined and bounded. Then $\left\{f_{k}\right\}_{k=1}^{\infty}$ is a Bessel sequence. 
Proof. By assumption, there exists $M>0$ such that

$$
\sum_{k=1}^{\infty}\left|\sum_{j=1}^{\infty} c_{j}\left\langle C f_{j}, U f_{k}\right\rangle\right|^{2} \leq M \sum_{k=1}^{\infty}\left|c_{k}\right|^{2}, \quad\left\{c_{k}\right\}_{k=1}^{\infty} \in \ell^{2}(\mathbb{N})
$$

Consider $\left\{c_{k}\right\}_{k=1}^{\infty}=(0, \ldots, 1,0, \ldots)$, we get

$$
\sum_{k=1}^{\infty}\left|\left\langle C f_{J}, U f_{k}\right\rangle\right|^{2} \leq M, \quad J \in \mathbb{N} .
$$

or

$$
\sum_{k=1}^{\infty}\left|\left\langle U^{*} C f_{J}, f_{k}\right\rangle\right|^{2} \leq M, \quad J \in \mathbb{N}
$$

Suppose that $\left\{f_{k}\right\}_{k=1}^{\infty}$ is not a Bessel sequence, then for all integer $N>0$ there exists $g_{N} \in H$ such that

$$
\sum_{k=1}^{\infty}\left|\left\langle g_{N}, f_{k}\right\rangle\right|^{2}>N\left\|g_{N}\right\|^{2}
$$

Therefore three cases may happen:

Case 1. If $g_{N} \in\left\{U^{*} C f_{k}\right\}_{k=1}^{\infty}$, then there exists $j \in \mathbb{N}$ such that $U^{*} C f_{j}=g_{N}$. Therefore by (2.5), we have

$$
\sum_{k=1}^{\infty}\left|\left\langle U^{*} C f_{j}, f_{k}\right\rangle\right|^{2}>N\left\|g_{N}\right\|^{2}
$$

which is a contradiction with (2.4).

Case 2. If $g_{N} \in \overline{\operatorname{span}}\left\{U^{*} C f_{k}\right\}_{k=1}^{\infty}$. Then

$$
\sum_{k=1}^{\infty}\left|\left\langle\sum_{j=1}^{\infty} c_{j} U^{*} C f_{j}, f_{k}\right\rangle\right|^{2}>N\left\|g_{N}\right\|^{2}
$$

or

$$
\sum_{k=1}^{\infty}\left|\sum_{j=1}^{\infty} c_{j}\left\langle C f_{j}, U f_{k}\right\rangle\right|^{2}>N\left\|g_{N}\right\|^{2},
$$

which is a contradiction with (2.2).

Case 3. If $g_{N} \notin \overline{\operatorname{span}}\left\{U^{*} C f_{k}\right\}_{k=1}^{\infty}$. Consider $M=\overline{\operatorname{span}}\left\{U^{*} C f_{k}\right\}_{k=1}^{\infty}$. Then, we can write $g_{N}=p_{N}+h_{N}$, where $p_{N} \in M$ and $h_{N} \in M^{\perp}, h_{N} \neq 0$, where $M^{\perp}$ is the orthogonal complement of $M$ in $H$. Now, we have

$$
\sum_{k=1}^{\infty}\left|\left\langle g_{N}, f_{k}\right\rangle\right|^{2}=\sum_{k=1}^{\infty}\left|\left\langle p_{N}+h_{N}, f_{k}\right\rangle\right|^{2}=\left.\sum_{k=1}^{\infty}\left\langle p_{N}, f_{k}\right\rangle\right|^{2}>N\left\|g_{N}\right\|^{2},
$$

Which is a contradiction like case 2. Therefore $\left\{f_{k}\right\}_{k=1}^{\infty}$ is a Bessel sequence.

Lemma 2.7. Let $\left\{f_{k}\right\}_{k=1}^{\infty}$ be a $(U, C)$-controlled Bessel sequence, then $\left\{f_{k}\right\}_{k=1}^{\infty}$ is a Bessel sequence in $\mathcal{H}$. 
Proof. Let $S_{U C}$ be the frame operator of $\left\{f_{k}\right\}_{k=1}^{\infty}$. Define $S_{F}=C^{-1} S_{U C}\left(U^{*}\right)^{-1}$. Since $S_{U C}, U$ and $C$ are bounded operators, $S_{F}$ is well-defined and bounded. Therefore, there exists $B>0$ such that

$$
\sum_{k=1}^{\infty}\left|\left\langle f, f_{k}\right\rangle\right|^{2}=\left\langle S_{F} f, f\right\rangle \leq B\|f\|^{2} .
$$

Definition 2.8. 13 Suppose that $E$ is an orthonormal basis for $H$. A bounded operator $T \in B(H)$ is called a Hilbert-Schmidt operator if

$$
\|T\|_{2}=\sqrt{\sum_{x \in E}\|T x\|^{2}}<\infty
$$

Definition 2.9. 13 Suppose that $E$ is an orthonormal basis for $H$. A bounded operator $T \in B(H)$ is called a trace-class operator if

$$
\|T\|_{1}=\sum_{x \in E}\langle|T|(x), x\rangle<\infty
$$

We denote the class of all Hilbert-Schmidt operators on $H$ and the class of trace-class operators on $H$ by $L^{2}(H)$ and $L^{1}(H)$, respectively. In [13, we see that $L^{1}(H) \subseteq L^{2}(H)$.

Theorem 2.10. (Polar Decomposition) 13 Let $V$ be a bounded linear operator on $H$. Then there is a unique partial isometry $U \in B(H)$ such that

$$
V=U|V|, \quad \operatorname{ker}(U)=\operatorname{ker}(V) .
$$

Moreover, $U^{*} V=|V|$.

Theorem 2.11. Suppose that $U, C \in G L(H)$. Let $F=\left\{f_{k}\right\}_{k=1}^{\infty}$ be a $(U, C)$ controlled frame and $G_{C U}$ is the $(U, C)$-controlled Gram operator associated to $\left\{f_{k}\right\}_{k=1}^{\infty}$. Then

(1) $G_{C U}$ is a Hilbert-Schmidt operator if and only if $H$ is finite dimensional.

(2) $G_{C U}$ is a trace-class operator if and only if $H$ is finite dimensional.

Proof. (1) Suppose that $\operatorname{dim} H<\infty$. By Lemma 2.7 $\left\{f_{k}\right\}_{k=1}^{\infty}$ is a Bessel sequence. Therefore there exists $B<\infty$ such that

$$
\begin{aligned}
\left\|G_{C U}\right\|_{2}^{2} & =\sum_{j=1}^{\infty} \sum_{k=1}^{\infty}\left|\left\langle C f_{j}, U f_{k}\right\rangle\right|^{2}=\sum_{j=1}^{\infty} \sum_{k=1}^{\infty}\left|\left\langle f_{j}, C^{*} U f_{k}\right\rangle\right|^{2} \\
(2.10) & =\sum_{k=1}^{\infty} \sum_{j=1}^{\infty}\left|\left\langle f_{j}, C^{*} U f_{k}\right\rangle\right|^{2} \leq B \sum_{k=1}^{\infty}\left\|C^{*} U f_{k}\right\|^{2} \leq B\left\|C^{*}\right\|^{2}\|U\|^{2} \sum_{k=1}^{\infty}\left\|f_{k}\right\|^{2} .
\end{aligned}
$$

Therefore by Proposition 5.1. in [1], $G_{C U}$ is a Hilbert-Schmidt operator. Now suppose that $G_{C U}$ is a Hilbert-Schmidt operator. By Proposition 2.2. $\left\{f_{k}\right\}_{k=1}^{\infty}$ is a frame for $H$, therefore there exists $A>0$ such that

$$
\begin{aligned}
A\left\|\left(C^{*} U\right)^{-1}\right\|^{-2} \sum_{k=1}^{\infty}\left\|f_{k}\right\|^{2} & \leq \sum_{k=1}^{\infty} \sum_{j=1}^{\infty}\left|\left\langle C f_{j}, U f_{k}\right\rangle\right|^{2} \\
& =\sum_{j=1}^{\infty} \sum_{k=1}^{\infty}\left|\left\langle C f_{j}, U f_{k}\right\rangle\right|^{2}=\left\|G_{C U}\right\|_{2}^{2} .
\end{aligned}
$$


The proof is evident by Proposition 5.1. in [1].

(2) Suppose that $\operatorname{dim} H<\infty$. By polar decomposition, there is a unique partial isometry $M \in B\left(\ell^{2}(\mathbb{N})\right)$ such that $\left|G_{C U}\right|=M^{*} G_{C U}$ and $G_{C U}=M\left|G_{C U}\right|$. Therefore

$$
\left|G_{C U}\right|=M^{*} T_{U F}^{*} T_{C F} .
$$

Now we show that $T_{C F}$ is a Hilbert-Schmidt operator. Suppose that $\left\{e_{k}\right\}_{k=1}^{\infty}$ is the canonical orthonormal basis for $\ell^{2}(\mathbb{N})$. So we have

$$
\left\|T_{C F}\right\|_{2}^{2}=\sum_{\left\{e_{k}\right\}_{k=1}^{\infty}}\left\|T_{C F}\left(e_{k}\right)\right\|^{2}=\sum_{k=1}^{\infty}\left\|C f_{k}\right\|^{2} \leq\|C\|^{2} \sum_{k=1}^{\infty}\left\|f_{k}\right\|^{2} .
$$

Therefore by Proposition 5.1. in [1, $T_{C F}$ is a Hilbert-Schmidt operator. Since $\left\|T_{U F}\right\|_{2}=\left\|T_{U F}^{*}\right\|_{2}$, we deduce that $T_{U F}^{*}$ is also a Hilbert-Schmidt operator. Now by Theorems 2.4.10. and 2.4.13. in [13] and (2.11), $\left|G_{C U}\right|$ is a trace-class operator. Since $G_{C U}=M\left|G_{C U}\right|$, by Theorem 2.4.15 in [13, $G_{C U}$ is a trace-class operator.

Vice versa, let $G_{C U}$ is a trace-class operator. Since $L^{1}(H) \subseteq L^{2}(H)$, we deduce that $G_{C U}$ is a Hilbert-Schmidt operator and so $H$ is a finite dimensional space by part (1).

Corollary 2.12. If $H$ is finite dimensional and $\left\{f_{k}\right\}_{k=1}^{\infty}$ is a $(U, C)$-controlled frame. Then $G_{C U}$ is a compact operator.

The following proposition gives a well-defined and bounded operator from $\ell^{1}(\mathbb{N})$ to $\ell^{\infty}(\mathbb{N})$ when the $(U, C)$-controlled Gram matrix is well-defined and bounded on $\ell^{2}(\mathbb{N})$.

Proposition 2.13. Suppose that the $(U, C)$-controlled Gram matrix associated to $\left\{f_{k}\right\}_{k=1}^{\infty}$, is well-defined and bounded. Then a bounded operator can be defined from $\ell^{1}(\mathbb{N})$ to $\ell^{\infty}(\mathbb{N})$.

Proof. Suppose that $G_{C U}$ is the operator associated to the matrix $\left\{\left\langle C f_{j}, U f_{k}\right\rangle\right\}_{k, j=1}^{\infty}$. Since $G_{C U}$ is well-defined and bounded on $\ell^{2}(\mathbb{N})$, for $\left\{c_{k}\right\}_{k=1}^{\infty} \in \ell^{2}(\mathbb{N})$, there exists $B>0$ such that

$$
\sum_{k=1}^{\infty}\left|\sum_{j=1}^{\infty} c_{j}\left\langle C f_{j}, U f_{k}\right\rangle\right|^{2} \leq B \sum_{k=1}^{\infty}\left|c_{k}\right|^{2}
$$

Therefore for each $j \in \mathbb{N}$,

$$
\sum_{k=1}^{\infty}\left|\left\langle C f_{j}, U f_{k}\right\rangle\right|^{2} \leq B
$$

Consider $M_{j, k}=\left\langle C f_{j}, U f_{k}\right\rangle$ and $M=\left\{M_{j, k}\right\}_{j, k=1}^{\infty}$. Then $M\left\{c_{k}\right\}_{k=1}^{\infty}=\left\{\sum_{j=1}^{\infty} M_{k, j} c_{j}\right\}_{k=1}^{\infty}$. Now, we show that $M$ defines a well-defined and bounded operator from $\ell^{1}(\mathbb{N})$ to $\ell^{\infty}(\mathbb{N})$.

First, we show that $\sum_{j=1}^{\infty} M_{k, j} c_{j}$ is convergent for each $k \in \mathbb{N}$. Given arbitrary $n, m \in \mathbb{N}, n \geq m$

$$
\left|\sum_{j=m+1}^{n} M_{k, j} c_{j}\right|^{2} \leq\left(\sum_{j=m+1}^{n}\left|M_{k, j}\right|\left|c_{j}\right|\right)^{2} \leq\left(\sum_{j=m+1}^{n}\left|M_{k, j}\right|^{2}\right)\left(\sum_{j=m+1}^{n}\left|c_{j}\right|^{2}\right) .
$$


By (2.13) and since $\ell^{1}(\mathbb{N}) \subseteq \ell^{2}(\mathbb{N})$, we get the result. Now, we show that $M$ is a bounded operator. For $\left\{c_{k}\right\}_{k=1}^{\infty} \in \ell^{1}(\mathbb{N})$, we have

$$
\begin{aligned}
\left\|M\left\{c_{k}\right\}_{k=1}^{\infty}\right\|_{\infty}^{2} & =\left\|\left\{\sum_{j=1}^{\infty} M_{k, j} c_{j}\right\}_{k=1}^{\infty}\right\|_{\infty}^{2}=\sup _{k \in \mathbb{N}}\left|\sum_{j=1}^{\infty} M_{k, j} c_{j}\right|^{2} \\
& \leq \sum_{j=1}^{\infty}\left|c_{j}\right|^{2} \sup _{k \in \mathbb{N}}\left(\sum_{j=1}^{\infty}\left|M_{k, j}\right|^{2}\right) \\
& \leq B \sum_{j=1}^{\infty}\left|c_{j}\right|^{2} \leq B \sum_{j=1}^{\infty}\left|c_{j}\right| .
\end{aligned}
$$

\section{3. $(U, C)$-CONTROLled Riesz Basis}

In this section, we propose a clear structure of $(U, C)$-controlled Riesz basis and show that every $(U, C)$-controlled Riesz basis is a $(U, C)$-controlled frame. Also an equivalent condition for a sequence $\left\{f_{k}\right\}_{k=1}^{\infty}$ being controlled Riesz basis given.

Definition 3.1. Suppose that $\left\{e_{k}\right\}_{k=1}^{\infty}$ is an orthonormal basis for $\mathcal{H}$. A $(U, C)$ controlled Riesz basis for $\mathcal{H}$ is a family of the form $\left\{U^{-1} C M e_{k}\right\}_{k=1}^{\infty}$, where $M$ is a bounded bijective operator on $H$.

Corollary 3.2. Every $(U, C)$-controlled Riesz basis is a Riesz basis for $H$.

Lemma 3.3. Suppose that $U$ is a positive invertible operator on a Hilbert space $H$. Then $U^{-1}$ is positive.

Proof. Since $U$ is an invertible operator, for each $x \in H$, there exists $y \in H$ such that $U y=x$. So

$$
\left\langle U^{-1} x, x\right\rangle=\left\langle U^{-1} U y, U y\right\rangle=\langle y, U y\rangle \geq 0
$$

Lemma 3.4. 7] If two bounded self-adjoint linear operators $S$ and $T$ on a Hilbert space $H$ are positive and commute, then their product $S T$ is positive.

Theorem 3.5. Suppose that $\left\{f_{k}\right\}_{k=1}^{\infty}$ is a $(U, C)$-controlled Riesz-basis for H. Assume that $U, C \in G L^{+}(H)$ and $U^{-1}$ and $C$ commute. Then $\left\{f_{k}\right\}_{k=1}^{\infty}$ is a $(U, C)$ controlled frame.

Proof. Since $\left\{f_{k}\right\}_{k=1}^{\infty}$ is a $(U, C)$-controlled Riesz-basis, for each $f \in H$, we have

$$
\begin{aligned}
\sum_{k=1}^{\infty}\left\langle f, U f_{k}\right\rangle\left\langle C f_{k}, f\right\rangle & =\sum_{k=1}^{\infty}\left\langle f, C M e_{k}\right\rangle\left\langle C U^{-1} C M e_{k}, f\right\rangle \\
& =\sum_{k=1}^{\infty}\left\langle f, C M e_{k}\right\rangle\left\langle C M e_{k},\left(U^{-1}\right)^{*} C^{*} f\right\rangle
\end{aligned}
$$


Consider $g_{k}=C M e_{k}$, for each $k \in \mathbb{N}$. Then $\left\{g_{k}\right\}_{k=1}^{\infty}$ is a Riesz basis for $H$. So by (3.1), we have

$$
\begin{aligned}
\sum_{k=1}^{\infty}\left\langle f, U f_{k}\right\rangle\left\langle C f_{k}, f\right\rangle & =\left\langle T_{g_{k}} T_{g_{k}}^{*} f,\left(U^{-1}\right)^{*} C^{*} f\right\rangle \\
& =\left\langle S_{g_{k}} f,\left(U^{-1}\right)^{*} C^{*} f\right\rangle \\
& =\left\langle C U^{-1} S_{g_{k}} f, f\right\rangle .
\end{aligned}
$$

Since $U, C \in G L^{+}(\mathcal{H})$ and $U^{-1}$ and $C$ commute by Lemma 3.3 and 3.4 , $C U^{-1} \in$ $G L^{+}(H)$. Since $S_{g_{k}} \in G L^{+}(H)$, by Proposition 2.3 there exist $A>0$ and $B<\infty$, such that $A I \leq C U^{-1} S_{g_{k}} \leq B I$.

Theorem 3.6. If $\left\{f_{k}\right\}_{k=1}^{\infty}$ is a $(U, C)$-controlled Riesz basis for $H$, then $\left\{f_{k}\right\}_{k=1}^{\infty}$ is a Bessel sequence. Furthermore, there exists a unique controlled Riesz basis sequence $\left\{g_{k}\right\}_{k=1}^{\infty}$ for $H$ such that for any $f \in \mathcal{H}$

(1) $f=\sum_{k=1}^{\infty}\left\langle f, g_{k}\right\rangle f_{k}=\sum_{k=1}^{\infty}\left\langle f, f_{k}\right\rangle g_{k}$.

(2) $f=\sum_{k=1}^{\infty}\left\langle f, U g_{k}\right\rangle C f_{k}=\sum_{k=1}^{\infty}\left\langle f, C f_{k}\right\rangle U g_{k}$.

Proof. (1) Suppose that $\left\{e_{k}\right\}_{k=1}^{\infty}$ is an orthonormal basis for $\mathcal{H}$. Since $\left\{f_{k}\right\}_{k=1}^{\infty}$ is a $(U, C)$-controlled Riesz basis, there exists a bounded bijective operator $M$ on $H$ such that $f_{k}=U^{-1} C M e_{k}$ for each $k \in \mathbb{N}$. So we have

$$
M^{-1} C^{-1} f=\sum_{k=1}^{\infty}\left\langle M^{-1} C^{-1} f, e_{k}\right\rangle e_{k}, \quad f \in \mathcal{H},
$$

so

$$
f=\sum_{k=1}^{\infty}\left\langle f,\left(C^{-1}\right)^{*}\left(M^{-1}\right)^{*} e_{k}\right\rangle C M e_{k}, \quad f \in \mathcal{H},
$$

therefore

$$
U^{-1} f=\sum_{k=1}^{\infty}\left\langle f,\left(C^{-1}\right)^{*}\left(M^{-1}\right)^{*} e_{k}\right\rangle U^{-1} C M e_{k}, \quad f \in \mathcal{H}
$$

and

$$
f=\sum_{k=1}^{\infty}\left\langle f, U^{*}\left(C^{-1}\right)^{*}\left(M^{-1}\right)^{*} e_{k}\right\rangle f_{k}, \quad f \in \mathcal{H} .
$$

Therefore by considering $g_{k}=U^{*}\left(C^{-1}\right)^{*}\left(M^{-1}\right)^{*} e_{k},\left\{g_{k}\right\}_{k=1}^{\infty}$ is a $\left(\left(U^{*}\right)^{-1},\left(C^{*}\right)^{-1}\right)$ controlled Riesz basis. A simple calculation shows that $\left\{g_{k}\right\}_{k=1}^{\infty}$ is a unique sequence that satisfies in (1).

(2) Considering $g_{k}=U^{-1}\left(C^{-1}\right)^{*} U^{*}\left(C^{-1}\right)^{*}\left(M^{-1}\right)^{*} e_{k}$, we get a unique $\left(C^{*} U, U^{*}\left(C^{-1}\right)^{*}\right)$ controlled Riesz basis, which satisfies in (2).

Corollary 3.7. If the sequences $\left\{f_{k}\right\}_{k=1}^{\infty}$ and $\left\{g_{k}\right\}_{k=1}^{\infty}$ satisfy in part (2) of Theorem 3.6. then $\left\langle C f_{k}, U g_{j}\right\rangle=\delta_{k, j}$.

Analogous to Theorem 3.6.6. of [ 6 , the following theorem gives an equivalent and practical condition for a sequence $\left\{f_{k}\right\}_{k=1}^{\infty}$ being a $(U, C)$-controlled Riesz basis.

Theorem 3.8. Suppose that $U, C \in G L^{+}(H)$. Assume that $U$ and $U^{-1}$ commute with $C$. For a sequence $\left\{f_{k}\right\}_{k=1}^{\infty}$ in $H$, the following conditions are equivalent:

(1) $\left\{f_{k}\right\}_{k=1}^{\infty}$ is a $(U, C)$-controlled Riesz basis for $H$. 
(2) $\left\{f_{k}\right\}_{k=1}^{\infty}$ is complete in $H$, and there exist constants $L, P>0$ such that for $\left\{c_{k}\right\}_{k=1}^{\infty} \in \ell^{2}(\mathbb{N})$ one has

$$
L \sum_{k=1}^{\infty}\left|c_{k}\right|^{2} \leq\left|\left\langle\sum_{k=1}^{\infty} c_{k} U f_{k}, \sum_{k=1}^{\infty} c_{k} C f_{k}\right\rangle\right| \leq P \sum_{k=1}^{\infty}\left|c_{k}\right|^{2} .
$$

Proof. (1) (1) $\Rightarrow(2)$. Assume that $\left\{f_{k}\right\}_{k=1}^{\infty}$ is a $(U, C)$-controlled Riesz basis, then there exists a bijective operator $M$ on $H$ such that $f_{k}=U^{-1} C M e_{k}$, for each $k \in \mathbb{N}$. By Theorem 3.6. $\left\{f_{k}\right\}_{k=1}^{\infty}$ is complete in $H$. For $\left\{c_{k}\right\}_{k=1}^{\infty} \in$ $\ell^{2}(\mathbb{N})$ we have

$$
\begin{aligned}
\left|\left\langle\sum_{k=1}^{\infty} c_{k} U f_{k}, \sum_{k=1}^{\infty} c_{k} C f_{k}\right\rangle\right| & =\left|\left\langle\sum_{k=1}^{\infty} c_{k} C M e_{k}, \sum_{k=1}^{\infty} c_{k} C U^{-1} C M e_{k}\right\rangle\right| \\
& =\left|\left\langle C M\left(\sum_{k=1}^{\infty} c_{k} e_{k}\right), C U^{-1} C M\left(\sum_{k=1}^{\infty} c_{k} e_{k}\right)\right\rangle\right| .
\end{aligned}
$$

By Lemma 3.3 and 3.4. $C U^{-1} \in G L^{+}(H)$. So there exist $A>0$ and $B<\infty$ such that

$$
\begin{aligned}
A\left\|C M\left(\sum_{k=1}^{\infty} c_{k} e_{k}\right)\right\|^{2} & \leq\left|\left\langle C M\left(\sum_{k=1}^{\infty} c_{k} e_{k}\right), C U^{-1} C M\left(\sum_{k=1}^{\infty} c_{k} e_{k}\right)\right\rangle\right| \\
& \leq B\left\|C M\left(\sum_{k=1}^{\infty} c_{k} e_{k}\right)\right\|^{2} .
\end{aligned}
$$

Since

$$
B\left\|C M\left(\sum_{k=1}^{\infty} c_{k} e_{k}\right)\right\|^{2} \leq B\|C M\|^{2} \sum_{k=1}^{\infty}\left|c_{k}\right|^{2},
$$

and

$$
A\left\|(C M)^{-1}\right\|^{-2} \sum_{k=1}^{\infty}\left|c_{k}\right|^{2}\|\leq\| C M\left(\sum_{k=1}^{\infty} c_{k} e_{k}\right) \|^{2},
$$

by (3.3) and (3.4), we deduce that

$$
A\left\|(C M)^{-1}\right\|^{-2} \sum_{k=1}^{\infty}\left|c_{k}\right|^{2} \leq\left|\left\langle\sum_{k=1}^{\infty} c_{k} U f_{k}, \sum_{k=1}^{\infty} c_{k} C f_{k}\right\rangle\right| \leq B\|C M\|^{2} \sum_{k=1}^{\infty}\left|c_{k}\right|^{2} .
$$

So considering $P=B\|C M\|^{2}$ and $L=A\left\|(C M)^{-1}\right\|^{-2}$, we get the proof.

(2) (2) $\Rightarrow(1)$ First, we show that $\left\{f_{k}\right\}_{k=1}^{\infty}$ is a Bessel sequence. For this since $C U \in G L^{+}(H)$, for $\left\{c_{k}\right\} \in \ell^{2}(\mathbb{N})$, we have

$$
\begin{aligned}
\left|\left\langle(C U)\left(\sum_{k=1}^{\infty} c_{k} f_{k}, \sum_{k=1}^{\infty} c_{k} f_{k}\right)\right\rangle\right| & =\left|\left\langle(C U)^{\frac{1}{2}}\left(\sum_{k=1}^{\infty} c_{k} f_{k}\right),(C U)^{\frac{1}{2}}\left(\sum_{k=1}^{\infty} c_{k} f_{k}\right)\right\rangle\right| \\
& =\left\|(C U)^{\frac{1}{2}}\left(\sum_{k=1}^{\infty} c_{k} f_{k}\right)\right\|^{2} .
\end{aligned}
$$


Now, we show that $\sum_{k=1}^{\infty} c_{k} f_{k}$ is convergent. Given arbitrary elements $m, n \in \mathbb{N}, n>m$, by (3.2) and (3.5), for $\left\{c_{k}\right\}_{k=1}^{\infty} \in \ell^{2}(\mathbb{N})$, we have

$$
\begin{aligned}
\left\|\sum_{k=m+1}^{n} c_{k} f_{k}\right\|^{2} & =\left\|(C U)^{\frac{-1}{2}}(C U)^{\frac{1}{2}}\left(\sum_{k=m+1}^{n} c_{k} f_{k}\right)\right\|^{2} \\
& \leq\left\|(C U)^{\frac{-1}{2}}\right\|^{2}\left\|(C U)^{\frac{1}{2}}\left(\sum_{k=m+1}^{n} c_{k} f_{k}\right)\right\|^{2} \\
& =\left\|(C U)^{\frac{-1}{2}}\right\|^{2} P \sum_{k=m+1}^{n}\left|c_{k}\right|^{2}
\end{aligned}
$$

Therefore $\sum_{k=1}^{\infty} c_{k} f_{k}$ is convergent and $\left\{f_{k}\right\}_{k=1}^{\infty}$ is a Bessel sequence and so $\left\{C^{-1} U f_{k}\right\}_{k=1}^{\infty}$ is a Bessel sequence. Choose an orthonormal basis $\left\{e_{k}\right\}_{k=1}^{\infty}$ for $H$, and extend by Lemma 3.3.6 in [7, the mapping $M e_{k}=C^{-1} U f_{k}$ to a bounded operator on $H$. In the same way, extend $V\left(C^{-1} U f_{k}\right)=e_{k}$ to a bounded operator on $H$. Then $M V=V M=I$, so $M$ is invertible, therefore $\left\{f_{k}\right\}_{k=1}^{\infty}$ is a $(U, C)$-controlled Riesz basis.

The following theorem gives a practical method to diagnose that $\left\{f_{k}\right\}_{k=1}^{\infty}$ is a $(U, C)$-controlled Riesz basis.

Theorem 3.9. Suppose that $U, C \in G L^{+}(H)$. Assume that $U$ and $U^{-1}$ commute with $C$. For a sequence $\left\{f_{k}\right\}_{k=1}^{\infty}$ in $H$, the following conditions are equivalent:

(1) $\left\{f_{k}\right\}_{k=1}^{\infty}$ is a $(U, C)$-controlled Riesz basis.

(2) $\left\{f_{k}\right\}_{k=1}^{\infty}$ is complete and it's controlled-Gram matrix $\left\{\left\langle C f_{k}, U f_{j}\right\rangle\right\}_{j, k=1}^{\infty}$ defines a bounded, invertible operator on $\ell^{2}(\mathbb{N})$.

(3) $\left\{f_{k}\right\}_{k=1}^{\infty}$ is complete, $(U, C)$-controlled Bessel sequence and has a complete biorthogonal sequence that is also a $(U, C)$-controlled Bessel sequence.

Proof. By a similar calculation of Theorem 3.4.4 in [7, Corollary 3.7] and Theorem 3.8, we get the proof.

\section{REFERENCES}

[1] P. Balazs, Frames and Finite Dimensionality: Frame Transformation, Classification and Algorithms, Appl. Math. Sci. 2(43), 2131-2144, (2008).

[2] P. Balazs, Hilbert-Schmidt operators and frames-classification, best approximation by multipliers and algorithms, Int. J. Wavelets Multiresolut. Inf. Process. 6(2), 315-330, (2008).

[3] P. Balazs, J -P. Antoine, A. Grybos, Weighted and controlled frames: mutual relationship and first numerical properties, Int. J. Wavelets Multiresolut. Inf. Process. 8, 109-132, (2010).

[4] I. Bogdanova, P. Vandergheynst, J. P. Antoine, L. Jacques, M. Morvidone, Stereographic wavelet frames on the sphere, Appl. Comput. Harmon. Anal. 16, 223-252, (2005).

[5] H. Bolcskei, F. Hlawatsch, H. G. Feichtinger, Frame-theoretic analysis of over-sampled filter banks, IEEE Trans. Signal process. 46(12), 3256-3268, (1998).

[6] O. Christensen, An Introduction to Frames and Riesz Bases, Boston, Birkhauser, 2003.

[7] O. Christensen, Frames and Bases, An Introductory Course, Boston, Birkhauser, 2008.

[8] I. Daubechies, A. Grossmann, Y. Meyer, Painless nonorthogonal expansions, J. Math. Phys. 27, 1271-1283, (1986).

[9] R. J. Duffin, A. C. Schaeffer, A class of nonharmonic Fourier series, Trans. Math. Soc. 72, 341-366, (1952).

[10] P. J. S. G. Ferreira, Mathematics for multimedia signal processing II: Discrete finite frames and signal reconstruction, In: Byrnes, J.s. (ed). signals processing for multimedia, PP. 35-54. IOS press, Amesterdam (1999). 
[11] D. Hua and Y. Huang, Controlled $K-g$-frames in Hilbert spaces, Results. Math., DOI 10.1007/s00025-016-0613-0, (2016).

[12] A. Khosravi and K. Musazadeh, Controlled fusion frames, Methods Funct. Anal. Topology, 18(3), 256-265 (2012).

[13] G. J. Murphy, $C^{*}$-algebras and operator theory, Academic Press, Inc., Boston, MA, 1990.

[14] K. Musazadeh, H. Khandani, Some results on controlled frames in Hilbert spaces, Acta Math. Sci. 36B(3), 655-665, (2016).

[15] E. Osgooei, A. Rahimi, Cross-Gram matrix associated to two sequences, preprint.

[16] E. Pekalska, R. P. W. Duin, The Dissimilarity Representation for Pattern Recognition: Foundations and Applications, World Scientific Publishing Co., Singapore, 2005.

[17] A. Rahimi and A. Fereydooni, Controlled $G$-frames and their $G$-multipliers in Hilbert spaces, An. St. Univ. Ovidius Constanta, 21(2), 223-236, (2013).

[18] A. Rahimi, S. Najafzadeh and M. Nouri, Controlled $K$-frames and their invariance under compact perturbation, (2016). arXiv:1602.03982

[19] M. Rashidi-Kouchi and A. Rahimi, On controlled frames in Hilbert $C^{*}$-modules, Int. J. Wavelets Multiresolut. Inf. Process., 15(4), 1750038, (2017).

[20] T. Strohmer, R. Jr. Heath, Grassmannian frames with applications to coding and communication, Appl. Comput. Harmon. Anal. 14(3), 257-275, (2003).

Faculty of sciences, Urmia University of Technology, Urmia, Iran.

E-mail address: e.osgooei@uut.ac.ir; osgooei@yahoo.com

Department of sciences, University of Maragheh, Maragheh, Iran.

E-mail address: rahimi@maragheh.ac.ir 